\title{
¿QUÉ HACEMOS CON LOS MIGRANTES? REPRESENTACIONES DE ALUMNOS DE ESCUELAS MEDIAS DE VILLA LUGANOY DISCURSOS POLÍTICOS SOBRE LA MIGRACIÓN LIMÍTROFE. BUENOS AIRES 2016-2018
}

\author{
Federico Luis ABIUSO ${ }^{1}$ \\ Gisele KLEIDERMACHER ${ }^{2}$ \\ Darío LANZETTA ${ }^{3}$
}

RESUMEN: El presente artículo surge como parte de los debates producidos en un grupo de investigación con sede en la Carrera de Sociología, Universidad de Buenos Aires, en el cual participamos. En base a cuestionarios aplicados en el marco de éste, a estudiantes de escuelas medias de Villa Lugano, Buenos Aires, en este escrito analizaremos aquellas preguntas que hacen referencia al acceso de migrantes a la ayuda social por parte del Estado. Paralelamente, se analizarán discursos políticos sobre la migración, que acompańaron cambios en la legislación migratoria argentina en los últimos años propiciando la sanción del DNU 70/2017 que modifica la Ley

\footnotetext{
1 Universidad de Buenos Aires (UBA), Buenos Aires - Argentina. Doctor en Ciencias Sociales y Licenciado en Sociología. Profesor en Metodología de la Investigación Social II (Carrera de Sociología). Becario Posdoctoral del Instituto de Humanidades y Ciencias Sociales del Litoral-Consejo Nacional de Investigaciones Científicas y Técnicas (IHUCSO LITORAL - CONICET). Orcid: http://orcid.org/0000-0001-5145-8837. abiusofederico@ yahoo.com.ar.

2 Universidad de Buenos Aires (UBA), Buenos Aires - Argentina. Doctora en Ciencias Sociales y Licenciada en Sociología. Investigadora Adjunta del Consejo Nacional de Investigaciones Científicas y Técnicas (CONICET) con sede en el Instituto de Investigaciones Gino Germani de la Facultad de Ciencias Sociales (UBA). Directora de Proyectos de Reconocimiento Institucional en Investigación (PRII) con sede en la Facultad de Ciencias Sociales (UBA) en temáticas relacionadas a las representaciones sociales, migraciones y discriminación en escuelas. Se desempeña actualmente como docente en grado y posgrado en la Facultad de Ciencias Sociales (UBA). Orcid: https://orcid.org/0000-0001-8739-8653. kleidermacher@gmail.com.

3 Universidad de Buenos Aires (UBA), Buenos Aires - Argentina. Becario doctoral y Licenciado en Sociología. Investiga temas relacionados al vínculo entre interculturalidad y clase social mediante el abordaje de representaciones sociales acerca de migrantes externos. Profesor en Metodología de la Investigación Social II (Carrera de Sociología). Orcid: https://orcid.org/0000-0002-0959-4453.dario_lanzetta@hotmail.com.
} 
¿Qué hacemos con los migrantes? Representaciones de alumnos de escuelas medias de villa lugano y discursos políticos sobre la migración limítrofe. Buenos Aires 2016-2018

Nacional de Migraciones 25.871, y que entendemos como expresión de un marco de situación contextual de conservadurismo político, habilitando un espacio discursivo de un conjunto de representaciones sociales, entre ellas, las de los estudiantes que aquí presentamos. Entre los principales hallazgos, se observa que casi un $40 \%$ de los alumnos encuestados no están de acuerdo con la provisión de ayuda a migrantes en forma de planes sociales o asignaciones por parte del Estado.

PALABRAS CLAVE: representaciones sociales; discursos políticos; migraciones; escuela.

\section{WHAT DO WE DO WITH MIGRANTS? REPRESENTATIONS OF HIGH SCHOOL STUDENTS IN VILLA LUGANO AND POLITICAL SPEECHES ON BORDER MIGRATION. BUENOS AIRES 2016-2018}

ABSTRACT: This paper arises as part of the debates produced in a research group based in the Sociology Career, University of Buenos Aires, in which we participate. Based on questionnaires applied to middle school students in Villa Lugano, Buenos Aires, in this paper we will analyze those questions that refer to the access of migrants to social assistance. At the same time, political discourses on migration will be analyzed, which accompanied changes in Argentine migration legislation in recent years, leading to the sanction of DNU 70/2017 that modifies the National Migration Law 25,871, and which we understand as an expression of a contextual situation framework of political conservatism, enabling a some speeches and social representations, among them, those of the students that we present here. Among the main findings, it is observed that almost $40 \%$ of the surveyed students do not agree with the provision of aid to migrants in the form of social plans or assignments by the State.

KEYWORDS: social representations; political speeches; migrations; school.

\section{"O QUE FAZEMOS COM OS MIGRANTES?" REPRESENTAÇÕES DE ALUNOS DO ENSINO MÉDIO EM VILLA LUGANO E DISCURSOS POLÍTICOS SOBRE A MIGRAÇÃO LIMÍTROFE. BUENOS AIRES 2016-2018}

RESUMO: Este artigo surge como parte dos debates produzidos em um grupo de pesquisa baseado na Curso de Sociologia da Universidade de Buenos Aires, do qual participamos. Com base em questionários aplicados neste âmbito a alunos do ensino médio em Villa Lugano, 
Buenos Aires, neste artigo analisaremos as questóes que se referem ao acesso dos migrantes à assistência social pelo Estado. Paralelamente, serão analisados os discursos políticos sobre migração, que acompanharam as mudanças na legislação migratória argentina nos últimos anos, levando à sanção do DNU 70/2017 que modifica a Lei Nacional de Migração 25.871, e que entendemos como expressão de um quadro de situação contextual do conservadorismo político, possibilitando um espaço discursivo de um conjunto de representaçôes sociais, entre elas, as dos alunos que aqui apresentamos. Dentre as principais constataçôes, observa-se que quase $40 \%$ dos alunos pesquisados não concordam com a prestação de auxílio aos migrantes na forma de planos sociais ou cessóes por parte do Estado.

PALAVRAS-CHAVE: representaçôes sociais; discursos politicos; migraçôes; escola.

\section{Introducción}

En el presente artículo nos proponemos realizar una aproximación a las representaciones sociales que producen estudiantes que asisten a escuelas medias de gestión pública en el barrio de Villa Lugano, ubicado en el sur de la CABA, respecto al acceso al derecho a ayudas sociales para personas migrantes. Entendemos que dichas representaciones no se producen en el vacío, sino que lo hacen en un contexto de conservadurismo político respecto a la gestión de las migraciones. Por contexto de conservadurismo político entendemos un panorama de auge de las políticas de derecha, donde la preferencia está en la moderación y en la prudencia más que en el cambio social, donde las referencias principales apuntan a actitudes culturales ligadas a la "familia", la "tradición" y la "nacionalidad". Asimismo, concebimos que se trata de un contexto de abierto rechazo al "populismo", tanto a sus estructuras como a sus protagonistas (siendo un ejemplo los sindicatos), así como de mitologización de la "república”. Inspirados en el análisis de las relaciones entre política y policía (AIMAR; GONZÁLEZ; MONTERO; SOZZO, 2005), tema en el que uno de los autores que suscribe a estas líneas se encuentra trabajando actualmente, diremos que más que pensar en una relación causa-efecto, de tipo lineal, entre los discursos políticos relevados y las representaciones producidas desde el ámbito educativo, aquellos discursos constituyen una condición de posibilidad habilitante de determinados universos de representaciones o núcleos representacionales, al decir de Néstor Cohen (2014), acerca del "otro". Ello apunta a la importancia de trabajar esta temática de representaciones acerca de acceso a derechos desde un "clima" de conservadurismo político, así como en un escenario de vulneración 
de derechos del colectivo migrante. Ambas dimensiones, discursos políticos y representaciones, serán desarrollados en los siguientes apartados del presente artículo. En cuanto a su estructura, iniciamos señalando características de la estrategia teórico-metodológica seleccionada (la implementación de una encuesta, técnica enmarcada en la investigación cuantitativa), así como las distintas decisiones involucradas en la experiencia de investigación cuyos resultados aquí presentamos. A continuación, damos cuenta de aquellos conceptos principales con los cuales interrogamos la base empírica. Los siguientes apartados se centran, por su parte y respectivamente, en distintos niveles de análisis: discursos políticos pronunciados durante la gestión de Cambiemos por parte de funcionarios de primera línea, reformas a la legislación migratoria acaecidas sobre todo a partir de la implementación del DNU 70/2017 y representaciones de la población estudiantil respecto al acceso al derecho a ayudas sociales para personas migrantes. Cierra el artículo una conclusión donde sintetizamos los principales resultados de la indagación efectuada, a la vez que señalamos un posible modo de articular los diferentes niveles de análisis mencionados.

\section{Metodología}

En este apartado presentamos una descripción de la estrategia teórico-metodológica para dar cuenta de las decisiones que se han ido tomando durante el proceso de investigación. Adoptamos como estrategia metodológica un enfoque cuantitativo con el objeto de trabajar con representaciones sociales, tomando en consideración que, la literatura metodológica suele recomendar optar por una línea cualitativa en ocasión de indagar acerca de los vínculos interculturales desde las representaciones sociales ${ }^{4}$. Sin desoír tales recomendaciones, entendemos que cada abordaje presenta sus propias complejidades, y que optar por uno u otro está relacionado con los propósitos y la estrategia de cada investigación en particular, en este caso, acceder a un universo amplio de estudiantes de escuelas públicas de nivel medio, para lo cual la encuesta 5 nos resultó más adecuada.

\footnotetext{
4 Cea D'Ancona y Valles (2010) apuntan a la dificultad respecto a la manifestación de representaciones acerca de migrantes, por lo que entienden que una perspectiva cualitativa favorecería develar lo que tiende a ocultarse. En este sentido, la entrevista en profundidad, sería acaso más adecuada puesto que entre sus potencialidades ofrecería la posibilidad de generar un marco de interacción propicio a establecer empatía entre entrevistador y entrevistado, a partir del cual este último pueda expresar con mayor libertad y en profundidad sus ideas (RUIZ OLABUÉNAGA, 1996; VALLES, 1997).

5 Nuestra estrategia se nutre no sólo de la intención de abordar un universo de estudio más amplio, sino que también el contar con experiencia de trabajo en investigaciones de corte cualitativo sobre la temática, despertó nuestro interés en reorientar el enfoque planteando asimismo un desafío de exploración teórico-metodológica.
} 
Tal como plantean algunos autores (MARRADI; ARCHENTI; PIOVANI, 2012), varias son las ventajas que proporciona esta técnica como, por ejemplo, la posibilidad de relevar múltiples propiedades referidas a muchos individuos, a fin de obtener información de distinto tipo, como puede ser respecto de opiniones, creencias y actitudes; sobre distintos temas, a partir de la aplicación de un cuestionario que puede ser administrado por encuestadores o distribuidos para su administración a la muestra diseñada para tal fin. Una de las desventajas que puede tener la técnica de encuesta está referida a la tasa de no respuesta. Contemplando esta posibilidad (MARRADI; ARCHENTI; PIOVANI, 2012), consideramos los distintos aspectos que podrían motivar la falta de participación por parte de los estudiantes. Entre ellos podían aparecer: la falta de interés en participar del estudio, cierta incomodidad o desacuerdo frente a la temática, o dificultades en la comprensión de alguna consigna del cuestionario. A fin de contrarrestar estas potenciales dificultades y evitar casos de no respuesta, nuestra estrategia fue la de concientizar a los estudiantes acerca de la importancia de sus respuestas y honestidad para los fines de la investigación y la calidad de los datos que se buscaban producir. Otras acciones implementadas para incrementar el número de casos de la muestra y reducir la tasa de no respuesta fueron la de aplicar las encuestas en horario escolar con el consentimiento de las instituciones y autoridades que nos dieron acceso. Además, nos permitieron presentar la actividad integrándola a los contenidos curriculares, dentro del marco de la institución en colaboración con un equipo de investigación ${ }^{6}$, aunque estaba exceptuado a participar el alumnado que no quisiera responder.

Es importante mencionar al respecto nuestra participación en investigaciones enmarcadas en proyectos anteriores, cuyos objetivos se centraron en conocer las representaciones sociales producidas al interior de las escuelas del AMBA y el poder judicial de la Nación. Entendemos que ello constituye una base de conocimiento previo sobre nuestro objeto de estudio y la posibilidad de reflexionar sobre modos posibles para su abordaje: Proyecto S007 (2008-2010), "Exclusión, control social y diversidad articulando la relación entre el migrante externo y las instituciones educativa y judicial"; Proyecto 20020100100040 (2011-2014), "Diversidad etno-nacional y construcción de desigualdades en las instituciones escolar y judicial. Un desafío teórico-metodológico en el abordaje de los casos del AMBA y la provincia de Mendoza" y Proyecto 20020100100040BA UBACYT (2014-1017), "Los puentes entre el poder judicial, la institución educativa y la sociedad civil ante la diversidad etno-nacional en el AMBA".

6 Cabe señalar que, dado que los sujetos objeto de nuestra investigación eran menores de edad, desde el comienzo de las tareas de campo se requirió proceder respetando procedimientos definidos para el caso. En este sentido, debimos primeramente contar con la aprobación del Comité de Ética del Instituto de Investigaciones Gino Germani, que dio consentimiento a nuestra tarea mediante una nota de autorización, la cual remitimos a cada una de las escuelas que accedieron a evaluar la propuesta para que realizáramos el relevamiento, adjuntando a la misma una copia del proyecto de investigación y del instrumento de registro. Evaluada y aprobada dicha instancia por parte de los directivos e inspectores de las escuelas, accedimos a las mismas en fecha acordada presentando una carta de aval y agradecimiento firmada por autoridades del instituto de investigaciones que consultamos para tal fin, y procedimos a realizar las tareas de campo. 
Con respecto a cómo medir adecuadamente la propia temática del estudio, coincidimos y por tanto tomamos como referencia para la fundamentación de nuestra propuesta a Cea D’Ancona (2009), por su experiencia en la medición acerca de problemáticas de racismo y xenofobia. Ella advierte sobre las dificultades de obtener, mediante declaraciones verbales, mediciones precisas, por el lugar de exposición en que se coloca al entrevistado a expresar consideraciones socialmente reprobables. Propone, en cambio, la modalidad de autocumplimentación por parte del encuestado, a fin de contribuir a obtener respuestas sinceras y menos condicionadas a lo socialmente deseables. Así es que implementamos dicha estrategia con el doble objetivo de, por un lado, disminuir el sesgo de deseabilidad social, pero también, una vez más, controlar la tasa de no respuesta. Para el caso de nuestra investigación, definimos la estrategia como "encuesta autoadministrada dirigida” en un contexto de grupo. La misma implicó que las personas encuestadas tuvieran a su cargo la tarea de responder al cuestionario, al mismo tiempo que la presencia de los encuestadores ofrecía la posibilidad de intervención en caso que fuera requerida alguna aclaración, pero también posibilitaba supervisar que los cuestionarios fueran respondidos adecuadamente, controlando de este modo la dinámica de la situación de encuesta durante el trabajo de campo (KLEIDERMACHER; LANZETTA, 2019). Las ventajas obtenidas a partir de la implementación de dicha estrategia fueron varias: la posibilidad de despejar dudas al momento de la aplicación del cuestionario, la posibilidad de controlar que fuera respondido por cada estudiante de manera individual, no vernos en la necesidad de restringir la extensión del cuestionario por alguna situación generada por incomodidad de las preguntas o por el tiempo de duración de aplicación del instrumento, y un factor nada menor, el beneficio de permitirnos acceder a un segmento poblacional de difícil acceso y concentrado espacialmente (KLEIDERMACHER; LANZETTA, 2019). La elección del barrio de Villa Lugano se funda en los datos proporcionados por el último Censo Nacional de Población del año 20107 , donde destaca la alta proporción de migrantes en la comuna donde dicho barrio se inserta. Tal como será desarrollado más extensamente en el apartado correspondiente, la población encuestada se compone mayoritariamente por estudiantes nativos ${ }^{8} \mathrm{y}$, en muy menor pro-

\footnotetext{
En la comuna 8, de la que forman parte los barrios de Villa Soldati, Villa Riachuelo y Villa Lugano, se destaca el elevado porcentaje de población originaria de Bolivia llegando a un $46,6 \%$ del total de extranjeros. En segundo orden se observa un $37,9 \%$ de personas de origen paraguayo, un $6,2 \%$ de no nativos de origen europeo, y una proporción de origen peruano del 5,2\% (INDEC, 2010).

8 "Toda persona que hubiera nacido y viva en Argentina. Esta segunda condición es importante porque remite a la posibilidad de participar en la red de relaciones interculturales y producir diferentes representaciones sociales acerca
} 
porción, por alumnos migrantes de origen boliviano, paraguayo y chileno ${ }^{9}$, que tenían a su arribo al país, entre 2 y 12 ańos. Por otro lado, otro de los criterios que nos orientaron a definir tal selección fueron las nociones de fragmentación y segmentación de las experiencias educativas (KESSLER, 2002). Sostenemos que es una manera posible, en una instancia inicial que pretendemos profundizar a futuro, de aproximarnos a la heterogeneidad de circuitos educativos existentes en la Ciudad Autónoma de Buenos Aires, diferenciados - según su matrícula en distintos estratos socioeconómicos de referencia. Referirnos al universo de estudio nos convoca a explicitar que implementamos un muestreo intencional que resultó en un total de 260 estudiantes. Tal como plantean algunos autores (COHEN; GÓMEZ ROJAS, 2019), este tipo de muestreo implica que el abordaje de los sujetos viene definido por criterios del investigador y la selección de estos se da en forma que la muestra sea lo más representativa a los efectos de la investigación que se pretende realizar. Aplicamos un cuestionario diseñado a partir de distintos bloques que incluyeron preguntas abiertas y cerradas, a partir de los cuales buscamos abordar distintas dimensiones representacionales de los estudiantes respecto a nativos y migrantes como así también acerca de relaciones de discriminación. Tomamos en consideración los recaudos sugeridos por Cea D’Ancona (2009), quien recomienda el uso de indicadores indirectos, en relación a sortear las dificultades al momento de medir la discriminación y la xenofobia. De este modo, entre otros varios aspectos, es que decidimos preguntar por la accesibilidad de derechos de los migrantes, como una forma indirecta de indagar en las representaciones que se tiene hacia ellos.

\section{Articulaciones teóricas}

Uno de los ejes de nuestra investigación se centra en la noción de representaciones sociales, las cuales comprendemos en el sentido que lo hace la psicología social, de la cual Serge Moscovici (1979) es su referente, esto es, como constructos cognitivos compartidos en la interacción social cotidiana que proveen a los individuos de un entendimiento de sentido común de sus experiencias en el mundo. Asimismo, Martín Mora (2002) agrega que estos sistemas de interpretación también guían las acciones. De esta manera, las representaciones sociales

de los migrantes externos. Se trata de una categoría heterogénea y compleja, discutible a partir de su enunciación" (COHEN, 2014, p.7).

9 Sin alcanzar en su totalidad al 13\% de la población encuestada, razón por la cual se decidió no dividir el análisis entre nativos y migrantes por ser tan pequeña la proporción de estos últimos. 
no quedan en un plano de las ideas, sino que se plasman también en los planos discursivos y actitudinales.

La selección de la institución escolar como ámbito desde donde realizamos el trabajo de campo, se debe a que, siguiendo a Cohen $(2009,2012)$ allí se llevan a cabo importantes acciones socializadoras en niños, niñas y adolescentes. De acuerdo con el autor, en la escuela y el poder judicial se materializa la acción pública sobre la población extranjera.

$\mathrm{Al}$ interior de ellas se definen y redefinen día a día los diferentes entramados sociales, los consensos y los conflictos entre unos y otros. El proceso de socialización en la escuela y el tratamiento (sanción) de los ilegalismos en el poder judicial, se constituyen y definen el lugar de cada uno, el lugar de los extranjeros y el lugar de los nativos (COHEN, 2012, p.187).

En relación a la edad, los y las jóvenes participantes de esta investigación se encuentran atravesando (o ya lo han hecho) lo que Gutton (1993) llamó "la pubertad". Es una etapa en la que entran en crisis las referencias identificatorias de la primera infancia, se cuestionan los ideales infantiles y las figuras significativas de la niñez y comienzan a conformarse nuevas subjetividades, siendo la escuela secundaria, en este caso, el ámbito privilegiado para aproximarse a estas representaciones sobre sí mismos, sobre los otros y sus derechos.

Nos interesa, en ese sentido, el análisis de la escuela, en tanto institución estatal que produce y reproduce determinadas imágenes sobre la migración. En este caso, tomamos las representaciones de los estudiantes que allí asisten y que han pasado por su proceso formador, sin por ello considerarlos actores pasivos receptores de discursos, sino que activamente los transforman/reproducen y/o producen nuevas formas representacionales sobre la migración, siendo algunas/ os de ellas/os también migrantes o formar parte de familias migrantes, tal como se analizará posteriormente.

Asimismo, entendemos que entre la escuela y la sociedad civil existen muros permeables, a través de los cuales se filtran representaciones sociales y discursos mediáticos y políticos que se filtran e ingresan a la institución (MALEGARIE; LANZETTA, 2013).

Cabe mencionar que tradicionalmente la intelectualidad y los políticos argentinos han procurado recibir inmigrantes europeos, desde los llamados realizados para poblar el suelo argentino de fines del siglo XIX, hasta la actual constitución nacional, que tras la reforma realizada en 1994, sigue contando 
con el llamado a ocupar el suelo por europeos ${ }^{10}$ (MARGULIS, 1997; VÉLEZ; MALUF, 2017, entre otros).

Es destacable esta preferencia por la población europea, porque tiene implicancias actuales, como es la invisibilización histórica de poblaciones de origen africano, afrodescendiente y originaria, así como también, su contracara, la no preferencia de migrantes de orígenes -entre otros- limítrofes, que portan rasgos originarios, asociados al atraso y la pobreza (CAGGIANO, 2005; ZEA, 1995; FRIGERIO, 2006; SEGATO, 2007).

Los medios masivos de comunicación y los discursos políticos, han contribuido a la conformación de un imaginario en relación a dicha migración, que, si bien siempre existente en la Argentina, aumentó su visibilización en la década del '90, debido a un doble proceso: su mayor presencia en las grandes ciudades, y el cambio de régimen de hipervisibilización de las diferencias étnicas en la década de los '90 (GRIMSON; BIDASEA, 2013). Durante esos años, los migrantes fueron culpabilizados de las altas tasas de desocupación que aquejaban al país debido a la instauración de un régimen neoliberal (CAGGIANO, 2015; GRIMSON; BIDASEA, 2013; NOVICK, 2012), así como del aumento de la delincuencia (COHEN, 2005; GALVANI, 2007; MONCLÚS MASÓ; GARCÍA, 2012) y la epidemia de cólera, entre otros males.

De esta forma, como analizan Domenech y Magliano (2008)exclusión y desigualdad, ya sean éstas de clase, etnia o género. En Argentina, los cambios en la conformación de los movimientos migratorios internacionales, en conjunto con las transformaciones sociales, políticas, económicas y culturales acontecidas en las últimas décadas, han plan-teado nuevos interrogantes acerca de la relación entre la desigualdad social y la diversidad cultural en contextos migratorios y multiculturales, especialmente aquellos caracterizados por la pobreza urbana. El presente trabajo refl exiona acerca de los discursos y las políticas de exclusión/ inclusión que desarrolla el Estado argentino con relación a la inmi-gración y los inmigrantes a partir de la década de los noventa, en el marco del auge neoliberal, y examina particularmente las representaciones y prácticas que se producen y refl ejan en el sistema educativo y en espacios escolares si-tuados en contextos marcados por la diversidad cultural, que contribuyen a la legitimación de la existencia y producción de la discriminación étnica y la 1 Docente-investigador del Centro de Estudios Avanzados de la Universidad Nacional de Córdoba (Argentina, los discursos y políticas referidos a la inmigración se han configurado en gran medi-

\footnotetext{
${ }^{10}$ Art. 25.- El Gobierno federal fomentará la inmigración europea; y no podrá restringir, limitar ni gravar con impuesto alguno la entrada en el territorio argentino de los extranjeros que traigan por objeto labrar la tierra, mejorar las industrias, e introducir y enseñar las ciencias y las artes.
} 
da a través de dos perspectivas, que coexisten en el imaginario social actual: los inmigrantes como "contribución" (es decir, los migrantes europeos) y los migrantes como "problema” o "amenaza" (es decir, los migrantes limítrofes y aquellos que no forman parte de la nación deseada). Al decir de los autores, "esto estableció la frontera que separa a aquellos deseables o indeseables, admitidos o rechazados, ya sea para ingresar al territorio o para formar parte de la nación" (DOMENECH; MAGLIANO, 2008, p.427).

En contextos de crisis económica y de revisión del Estado de Bienestar es cuando más se percibe la inmigración como "amenaza" y se refuerza la idea de que los derechos sociales han de restringirse en los inmigrantes y primarse en los autóctonos. En tales escenarios:

La discriminación positiva a favor de los inmigrantes (para ayudar a su integración) se considera, por una parte de la población que no se considera racista, un 'agravio comparativo'. Defienden el principio de la 'prioridad' en el acceso a los recursos para los 'nacionales'. Este discurso es característico de las nuevas manifestaciones de racismo y xenofobia (CEA D’ANCONA, 2009, p.203).

Pensamos que tanto los discursos políticos como las representaciones de los estudiantes, contienen elementos discriminatorios, entendiendo por discriminación una distinción ideológica que infravalora a los diferentes mediante el rechazo y la intolerancia (RIVERO SIERRA, 2011). En la escuela estas cuestiones toman mayor dimensión, debido al tratamiento de la diversidad que allí se realiza, que muta en ocasiones en relaciones de desigualdad, tanto por los estereotipos de algunos docentes, como por la forma en que se trata esta diversidad cultural, ya sea exotizándola o bien desvalorizándola (NOVARO; DIEZ, 2011).

Si bien no será abordado en este trabajo, hemos analizado en otros escritos las representaciones sociales discriminatorias de adolescentes en las escuelas, siguiendo estudios clásicos en la temática como los de Sinisi (1998), Kornblit (2008), Novaro y Diez (2011), Beheran (2012), entre muchos otros ${ }^{11}$.

\footnotetext{
${ }_{11}$ Al respecto, existen otras investigaciones que abordaron las concepciones en torno al derecho de niños y jóvenes en el contexto escolar y desde otras disciplinas distintas a la sociológica. Ilustrativamente, podemos referir a la tesis de Laura Martínez (2017).
} 


\section{"No podemos permitir que el crimen siga eligiendo a la Argentina como un lugar para venir a delinquir". Discursos políticos sobre la migración}

Tal como señaláramos en la introducción, las representaciones, objeto de nuestro análisis, se producen en un contexto de conservadurismo político respecto a la gestión de las migraciones. En este sentido, consideramos significativo describir distintos discursos políticos pronunciados durante la gestión de Cambiemos por parte de funcionarios de primera línea, concibiendo, como uno de los modos de articulación entre discursos y representaciones - el cual pretendemos profundizar a futuro - que tales discursos constituyen una condición de posibilidad habilitante de determinados universos de representaciones o núcleos representacionales, al decir de Cohen (2014), acerca del "otro".

Como iniciativa impulsada a partir del pánico moral desatado por el asesinato de Brian Aguinaco ${ }^{12}$ - por obra de un adolescente hijo de padres peruanos - se instrumentaron distintos cambios en la política migratoria durante el gobierno presidido por Mauricio Macri (2015-2019), algunos de los cuales impactaron en mayores niveles de punitividad para con la población migrante; entendemos por punitividad el nivel de dolor o sufrimiento producido por el sistema penal (SOZZO, 2017). Ahora bien, ¿cuáles fueron tales medidas instrumentadas y cuáles fueron los discursos que, pronunciados por distintos funcionarios del gobierno nacional, los acompañaron ${ }^{13}$ Una de ellas se remonta a una cuestión de larga data desde la perspectiva policial, criminológica e incluso estatal, el imperativo por identificar a los "sospechosos" (GARCÍA FERRARI, 2010).

Más concretamente, podemos referir a la implementación del sistema de Información Anticipada de Pasajeros (API, por sus siglas en ingles), como una iniciativa orientada a que las aerolíneas puedan brindar datos sobre antecedentes delictivos de los viajeros que quieran ingresar a Argentina para así "impedir el ingreso de delincuentes"14. Para Rogelio Frigerio, en ese entonces ministro del

\footnotetext{
12 Para mayor información de este caso, puede consultarse: https://www.clarin.com/policiales/hoy-dieron-perpetuamotochorros-crimen-brian-aguinaco_0_RpbEy-PfG.html. Acceso en: 25 ago. 2021.

${ }_{13}$ Para el relevamiento de las medidas impulsadas, así como para el grillado de los discursos de funcionarios de gobierno que las acompañaron y propiciaron, fueron consultadas noticias periodísticas de los diarios La Nación y Clarín, específicamente en sus versiones digitales, editadas entre los años 2015 y 2019.

14 Anuncio del Ministerio del Interior. Control migratorio: las aerolíneas deben anticipar datos de los pasajeros, Clarín, 25/01/2017. Disponible en: https:/www.clarin.com/politica/control-migratorio-aerolineas-deben-anticipardatos-pasajeros_0_B19K_hUDx.html. Acceso en: 26 ago. 2021.
} 
Interior, "la medida tiene más que ver con la política de la administración de Mauricio Macri contra la delincuencia que con la migratoria” ${ }^{15}$.

Es relevante destacar que esta clase de iniciativas se presentan, en ocasiones, en conjunción con un léxico asociado a la noción de riesgo, cuya importancia en las estrategias de control contemporáneas ha sido trabajada por distintos autores, provenientes de diversas regiones geográficas (GARLAND, 1997; DE GIORGI, 2005, 2006; O’MALLEY, 2006). Al respecto, Horacio García, quien al momento se desempeñaba como Director Nacional de Migraciones, señaló que la implementación del API contribuiría a la seguridad, pues al cruzar diversos datos de cada pasajero se podrían "trazar perfiles de riesgo" asociados a delitos específicos como narcotráfico y trata de personas.

Incluso, en torno al mencionado imperativo por identificar, el propio Macri destacó - en el contexto de la primera conferencia de prensa realizada durante el año 2017 - que "no podemos permitir que el crimen siga eligiendo a la Argentina como un lugar para venir a delinquir. Necesitamos articular con los demás países para saber quién es quién". Sobre la misma temática, Patricia Bullrich, a cargo del Ministerio de Seguridad, señalaba: "Una persona que tiene antecedentes en su país, no puede entrar a la Argentina. Por eso queremos tener una base de datos para poder analizar quién es el que llega, especialmente con aquellos países cuyo volumen de migración es importante" 16 .

En sintonía, otro de los cambios acaecidos respecto a los controles migratorios apunta a la creación de la Comisión Nacional de Fronteras, oficializada por medio del decreto 68/2017. Firmado por Macri, el jefe de Gabinete Marcos Peña y los ministros Frigerio (Interior) y Bullrich (Seguridad). El organismo se presentaba como parte de una estrategia integral de lucha contra el narcotráfico, la trata de personas, el contrabando y otros delitos transnacionales ${ }^{17}$.

El mismo día de publicación del decreto en el Boletín Oficial, Frigerio destacó - en una entrevista con Radio La Red -, el siguiente estado de situación acerca de los "delincuentes" que ingresan al país: "Argentina seguirá siendo abierta para aquellos que quieran venir a formar parte de nuestra comunidad y

\footnotetext{
15 Seguridad fronteriza. Arrancan los nuevos controles migratorios para evitar el ingreso al país de personas con antecedentes, Clarín, 25/01/2017. Disponible en: https:/www.clarin.com/politica/arrancan-nuevos-controlesmigratorios-evitar-ingreso-pais-personas-antecedentes_0_BJr1bo8wl.html. Acceso en: 26 ago. 2021.

${ }^{16}$ Las palabras entrecomilladas incluidas en este párrafo son extraídas de El endurecimiento de las fronteras, Clarín, 17/01/2017. Disponible en: https:/www.clarin.com/politica/macri-favor-controles-migratorios-necesitamossaber_0_r1BqbniUx.html. Acceso en: 26 ago. 2021.

${ }_{17}$ Para mayor detalle, el texto del decreto puede consultarse desde la página web de InfoLEG: http://servicios. infoleg.gob.ar/infolegInternet/anexos/270000-274999/271198/norma.htm. Acceso en: 25 ago. 2021.
} 
contribuir con el crecimiento de la argentina, pero inflexibles para los delincuentes que quieran venir a nuestro país"18.

En un contexto de mayores controles en las fronteras ${ }^{19}$, no resulta tampoco sorpresivo ni chocante que se le haya dado prensa a operativos por parte de la Policía Federal Argentina, como indicador de que "algo se está haciendo" para combatir al delito ${ }^{20}$. Especificando, en ese entonces, cuáles eran los tipos de delitos a los cuales se buscaba hacer frente, Bullrich sostuvo, "nuestro problema con la frontera no es la gente, nosotros no tenemos un problema con la inmigración, tenemos un problema con el narcotráfico y con el contrabando" 21.

Es relevante destacar que, en reiteradas ocasiones, los discursos gubernamentales en que se hacían públicos los vínculos entre inmigración y delincuencia eran acompañados por un recurso al "lenguaje de los números" en torno a la criminalidad (SOZZO, 2003), a saber: estadísticas penitenciarias "oficiales" que podrían desglosarse en porcentaje de presos detenidos en cárceles federales y cifras sobre migración y delito. Si bien no es un recurso novedoso, es importante señalar que es convocado a sustentar, con frecuencia, aquellos discursos que vinculan entre sí a la inmigración con la delincuencia (ABIUSO, 2020). Cabe hacer notar también que en ocasiones se alzaron voces, desde distintos ámbitos, cuestionando tal proceder señalando que se trataba de información descontextualizada ${ }^{22}$.

Continuando nuestro recorrido por las distintas medidas implementadas, el panorama de endurecimiento de los controles, así como de intersección entre política migratoria y política criminal, encuentran su punto más álgido en la

\footnotetext{
${ }^{18}$ Frigerio: "Queremos evitar que ingresen delincuentes y acelerar los trámites para expulsarlos; no pasa de ahí", La Nación, 26/01/2017. Disponible en: https://www.lanacion.com.ar/politica/frigerio-queremos-evitar-que-ingresendelincuentes-y-acelerar-los-tramites-para-expulsarlos-no-pasa-de-ahi-nid1979151/. Acceso en: 26 ago. 2021.

19 Como marco del "efecto-Trump", otras de las iniciativas que fueron pensadas desde la Casa Rosada fueron la creación de un "fuero migratorio" que atienda exclusivamente asuntos de extranjería y de una policía migratoria, con la cual se pretendía imitar a la US Border Patrol de EEUU y a las de otros países tales como Ecuador, Panamá y Costa Rica.

${ }^{20}$ Ver ilustrativamente En fotos: el operativo sorpresa en la terminal de micros de Liniers, La Nación, 27/01/2017. Disponible en: https:/www.lanacion.com.ar/seguridad/en-fotos-el-operativo-sorpresa-en-la-terminal-de-microsde-liniers-nid1979629/. Acceso en: 26 ago. 2021.

21 Patricia Bullrich: "No barajamos en absoluto la construcción de un muro con Bolivia", El Tribuno, 29/01/2017. Disponibleen https:/www.eltribuno.com/salta/nota/2017-1-29-1-30-0-patricia-bullrich-no-barajamos-en-absolutola-construccion-de-un-muro-con-bolivia. Acceso en: 26 ago. 2021.

22 Al respecto, podrían mencionarse el trabajo de Marta Monclús Masó (2017), así como también desde un ámbito distinto - el periodístico - se podría destacar la nota de Sol Amaya, editada en La Nación el 27 de febrero de 2018 y titulada, "La lupa sobre los inmigrantes: ¿qué impacto real tienen sobre la Argentina?", donde se incluye un apartado titulado "Los inmigrantes y el delito".
} 
implementación del DNU 70/2017²3, a partir del cual el gobierno nacional formalizó cambios - de forma unilateral por el Poder Ejecutivo- en la Ley de Migraciones 25.871. Desarrollaremos los principales puntos del decreto en el siguiente apartado.

\section{Cambios y continuidades en la legislación migratoria argentina. Visiones de Estado acerca de la cuestión migratoria como cuestión criminal}

La historia de las migraciones en Argentina estuvo atravesada por distintas iniciativas normativas por canalizar, direccionar y regular los flujos migratorios internacionales, ya sea que se trate de mecanismos restrictivos o estrategias de control más sutiles, las cuales podríamos definir, siguiendo a Domenech (2013), como políticas de "control con rostro humano". La formulación de estas iniciativas no estuvo exenta de tensiones y conflictos entre distintos actores, y el DNU 70/2017 no constituye una excepción al respecto.

El decreto, que contó con el aval de funcionarios de distintas áreas de gobierno, así como con el apoyo de referentes de la oposición ${ }^{24}$, fue objeto de comentarios críticos por parte de diversos organismos de derechos humanos (CELS, CAREF, IARPIDI, entre otros) e investigadores pertenecientes al campo científico-académico (MONCLÚS MASÓ, 2017; ZAYAT, 2017; CANELO; GAVAZZO; NEJAMKIS, 2018; PENCHASZADEH; GARCÍA, 2018-2019; TEDESCO; KLEIDERMACHER; GRAZZIOTIN NOSCHANG, 2019). Al respecto, en el pronunciamiento de la Comisión Interamericana de Derechos Humanos (CIDH), ante la "defensa" del decreto por parte de autoridades del gobierno, este fue considerado como una "regresión" por parte de Margarette May Macaulay, a cargo de la Relatoría sobre los Derechos de los Migrantes de la $\mathrm{CIDH}^{25}$.

El DNU 70/2017 fue presentado como parte de una política de lucha contra el "crimen organizado internacional" y/o delitos vinculados a la "narcocriminalidad" 26 , al ser referidos el incremento de los presos extranjeros en cárceles

\footnotetext{
${ }^{23}$ Para mayor detalle del mismo, remitimos a http://www.migraciones.gov.ar/pdf_varios/residencias/Decreto_ 70-2017.pdf. Acceso en: 25 ago. 2021.

${ }^{24}$ Pudiéndose mencionar, al respecto, los casos de Miguel Ángel Pichetto (PJ Federal) y Sergio Massa (Frente Renovador).

${ }^{25}$ La CIDH consideró que el decreto migratorio de Macri es una "regresión", La Nación, 23/03/2017. Disponible en: https://www.lanacion.com.ar/politica/la-cidh-considero-que-el-decreto-migratorio-de-macri-es-una-regresionnid1997618/. Acceso en: 26 ago. 2021.

${ }^{26}$ Controles más estrictos Mauricio Macri firmó el decreto que modifica la Ley de Migraciones: los principales puntos, Clarín, 30/01/2017. Disponible en: https:/www.clarin.com/politica/mauricio-macri-firmo-decreto-modifica-
} 
federales y la incidencia de éstos en aquellos detenidos por delitos de drogas; explicitando, de tal forma, una identificación entre inmigración y delito.

De manera desagregada, entre los cambios que introdujo en la legislación vigente, podemos mencionar la modificación e incorporación de nuevas causales de impedimento de ingreso y permanencia en el país, así como para la cancelación de la residencia, ya que a partir del decreto puede rechazarse o cancelarse la residencia de cualquier persona que tenga un conflicto con la ley penal, sin una consideración explicita de la gravedad del delito, y aun en los casos en que no haya una sentencia firme.

Otro de los cambios apunta a la introducción de un procedimiento migratorio especial sumarísimo para la detención y expulsión de migrantes, el cual se caracteriza por la reducción de los plazos en su tramitación, tanto del procedimiento administrativo como de los recursos judiciales.

Ambos puntos, controles más estrictos para el ingreso y permanencia de extranjeros en el país, así como una pretendida agilización y aceleración en la expulsión en el caso de aquellos que cometen delitos, son elocuentemente sintetizados por Frigerio, al destacar que "debemos ser muy restrictivos con los que quieran venir a delinquir o quienes hayan delinquido en Argentina" 27.

Otros de los efectos nocivos impulsados por el decreto apuntan a la vulneración de derechos que se encontraban reconocidos en la ley migratoria 25.871; concretamente, podemos referir a la modificación del art. 70 acerca del derecho de defensa del migrante, así como a las nuevas previsiones en el art. 86 en torno al acceso a la justicia y a la asistencia jurídica gratuita.

Retomando, a su vez, lo trabajado por distintos investigadores de la temática (GALOPPO, 2017; CANELO; GAVAZZO; NEJAMKIS, 2018), diremos que nos interesa recuperar también aquí el efecto social producido por el DNU 70/2017; al darle sustento a la asociación entre inmigración y delincuencia, y generando un consenso social en torno a la legitimación de ese tipo de medidas.

Es en el trasfondo de estos cambios, en el contexto de proliferación de discursos gubernamentales, que hacían pública la relación entre inmigración, delincuencia e (in)seguridad ${ }^{28}$ y de prácticas que significaban un retroceso en la política migratoria y vulneración de los derechos de los migrantes que viven en

\footnotetext{
ley-migraciones-principales-puntos_0_ByiU5KnDg.html. Acceso en: 26 ago. 2021.

${ }_{27}$ Tenemos bastante con nuestros delincuentes, La Nación, 31/07/2017. Disponible en: https://www.lanacion.com. ar/politica/tenemos-bastante-con-nuestros-delincuentes-nid1980390/. Acceso en: 26 ago. 2021.

${ }^{28}$ Podemos reconocer distintos antecedentes históricos de este vínculo, uno de ellos relacionado a las leyes y decretos que, impulsados desde la década de 1960, estuvieron orientados a la construcción del migrante limítrofe como "amenaza" a la seguridad (DOMENECH, 2011a, 2011b; PEREIRA, 2016; ABIUSO, 2020). Otro antecedente, más reciente en el tiempo, gira en torno a la "toma" del Parque Indoamericano en 2010 y los discursos de
} 
la Argentina ${ }^{29}$. Es un contexto que podríamos pensar, a su vez siguiendo a distintos autores, a partir de la noción de "crimigración" ${ }^{30}$, desde donde nos interesa adentrarnos en un dispositivo institucional en particular, la institución escolar.

Más específicamente, proponemos indagar en las representaciones que tienen los estudiantes acerca del acceso (diferencial o no) a derechos, para las colectividades que fueron seleccionadas en nuestra experiencia de investigación, en el marco de un panorama de conservadurismo político y vulneración de derechos del colectivo migrante.

En otros escritos, hemos abordado las representaciones sociales que los estudiantes construyen respecto a diversas nacionalidades migrantes, sin embargo, en esta oportunidad, nos propusimos analizar las representaciones respecto al acceso a ayudas sociales del estado para población de origen migrante, entendiendo que se trata de una forma de analizar la visión que sobre las migraciones tienen los estudiantes de las escuelas analizadas. Es decir, su acuerdo o no con la recepción de ayudas sociales por parte de los migrantes, es una forma también de ver a quienes se representa formando parte de la nación y a quienes no, así como también, determinadas representaciones que se construyen acerca de diversas nacionalidades.

El presente artículo ha sido escrito durante la pandemia de COVID-19, pero trabajando con datos producidos con anterioridad a la misma. Si bien no forma parte del núcleo de nuestra investigación, proponemos a modo de reflexión, considerar que la condición de vulnerabilidad económica y social del colectivo migrante se ha profundizado aún más en el contexto que nos toca transitar a partir de la pandemia COVID-19. En este marco, el gobierno nacional ha dictado el Aislamiento Social Preventivo y Obligatorio (ASPO), condicionando mayormente la posibilidad de generar ingresos a quienes estás insertos en el sector informal en el mercado de trabajo, que es en donde suele insertarse este colectivo. Proponemos considerar, acaso, que la situación que describimos en nuestro estudio a partir del análisis de los datos presentados puede ser un aporte a una lectura crítica del presente.

funcionarios del Gobierno de la Ciudad de Buenos Aires -incluido, entre ellos, el propio Macri- acerca de la "inmigración descontrolada" (CANELO, 2015).

${ }^{29}$ Agréguese la iniciativa conjunta de la Dirección Nacional de Migraciones, el Ministerio de Seguridad y el Ministerio de Justicia de la Ciudad de Buenos Aires para crear un Centro de Detención de Migrantes.

${ }^{30}$ En tal sentido, han proliferado en los últimos años investigaciones empíricas en torno a la interrelación entre control migratorio y sistema penal, uno de cuyos aspectos refiere, siguiendo a Brandariz García, Dufraix y Quinteros (2018), al carácter materialmente punitivo de los instrumentos de gobierno de fronteras, como el internamiento o las expulsiones de extranjeros. 


\section{"Ayuda sociales a migrantes ¿si o no? Representaciones de los alumnos de escuelas de Villa Lugano}

En el apartado a continuación, presentaremos las representaciones de la población estudiantil que asiste a tres escuelas medias del barrio de Villa Lugano, ubicado en el sur de la Ciudad Autónoma de Buenos Aires. Sin embargo, en primer lugar, consideramos importante caracterizar a la población a la cual nos referimos.

Gráfico 1 - Estudiantes de escuelas Medias de Villa Lugano por lugar de Nacimiento. Ciudad Autónoma de Buenos Aires, 2017.

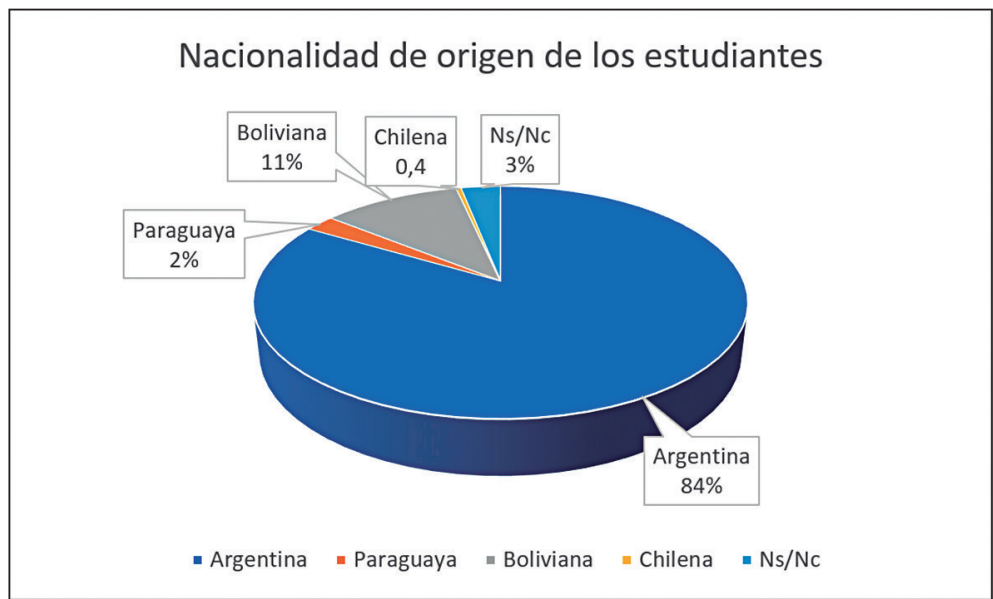

Fuente: Producción propia en base a datos construidos por el equipo de Investigación del Proyecto de Reconocimiento Institucional "Representaciones sociales de jóvenes y adultos hacia migrantes bolivianos, paraguayos, asiáticos y africanos en "barrios sur" de CABA. 2014-2016"

Tan como se desprende del gráfico $\mathrm{N}^{\circ} 1$, de un total de 260 estudiantes que conformaron la muestra, la mayor parte de la población puede ser considerada nativa, entendiendo por tal categoría, "toda persona que hubiera nacido y viva en Argentina” (COHEN, 2014, p.7) ${ }^{31}$, como adelantáramos en el apartado metodológico. Dicha categoría concentra al $84 \%$ de la población. Los y las estudiantes nacidos en Bolivia representan al 11\%, siguiendo la población nacida en Paraguay con un 2\% los nacidos en Chile que representan apenas el 0,4\%.

\footnotetext{
31 Tal como destaca Cohen (2014), se trata de una categoría heterogénea y compleja, discutible a partir de su enunciación.
} 
De esta forma, se observa que la población estudiantil nacida en Bolivia es la más numerosa luego de la argentina, siendo muy minoritarias las otras nacionalidades limítrofes. Debe tenerse en cuenta que aquellos estudiantes nacidos en dichos países, han llegado a la Argentina a edades muy tempranas, teniendo entre dos y doce años al momento de arribo. Si bien sus trayectorias de vida pueden incidir en sus representaciones, en este estudio no serán analizadas de manera diferencial.

Con relación a las representaciones sociales a las que aquí referimos constitutivas de la dimensión "acceso a derechos", las mismas parten de un bloque ubicado promediando el cuestionario. En ese sentido, resulta importante mencionar que el cuestionario cuenta con diversos bloques de preguntas, donde se indaga por las representaciones que los estudiantes construyen sobre diversos grupos migratorios, sobre la identidad nacional, así como las relaciones que se construyen en el aula en relación a la migración, a temáticas de discriminación, entre otras. En este caso, las preguntas que fueron tomadas para el análisis, forman parte de un bloque donde se indaga respecto a la identidad nacional y los países a los cuales "debería imitarse". En concreto, las preguntas aquí analizadas han sido formuladas de la siguiente forma: ¿Estás de acuerdo en que las personas de otras nacionalidades que viven en la Argentina reciban programas o ayudas sociales del gobierno? ¿Por qué?

Tabla 1 - Estudiantes de escuelas Medias de Villa Lugano según acuerdo con que migrantes reciban programas o ayudas sociales. Ciudad Autónoma de Buenos Aires, 2017.

\begin{tabular}{|c|c|c|}
\hline & Frecuencia & Porcentaje \\
\hline Sí & 160 & 61,5 \\
\hline No & 95 & 36,5 \\
\hline $\mathrm{Ns} / \mathrm{Nc}_{\mathrm{c}}$ & 5 & 1,9 \\
\hline Total & 260 & 100 \\
\hline
\end{tabular}

Fuente: Producción propia en base a datos construidos por el equipo de Investigación del Proyecto de Reconocimiento Institucional "Representaciones sociales de jóvenes y adultos hacia migrantes bolivianos, paraguayos, asiáticos y africanos en "barrios sur" de CABA. 2016-2018"

De la tabla $\mathrm{N}^{\circ} 1$ se desprende que el $61,5 \%$ de la población encuestada estaría de acuerdo con que el gobierno brinde ayuda o programas sociales a población de origen migrante, mientras que el $36,5 \%$ no lo estaría. 
Al preguntar los motivos por los cuales estaría de acuerdo con que se brinde dicha ayuda, hemos realizado un procedimiento que implicó el cierre de respuestas y el armado de categorías, dando como resultado la tabla que se presenta a continuación:

Tabla 2 - Estudiantes de escuelas Medias de Villa Lugano según motivo de acuerdo con que migrantes reciban programas o ayudas sociales. Ciudad Autónoma de Buenos Aires, 2017.

\begin{tabular}{|l|r|r|}
\hline \multicolumn{1}{|c|}{ Motivo } & f & \multicolumn{1}{c|}{$\%$} \\
\hline Necesidad y nuevo comienzo & 63 & $38,20 \%$ \\
\hline Personas con igualdad de derechos & 60 & $36,40 \%$ \\
\hline Acepta con restricción & 15 & $9,10 \%$ \\
\hline Políticamente correcto & 8 & $4,80 \%$ \\
\hline Contribuyen al país & 4 & $2,40 \%$ \\
\hline No sabe/No contesta & 15 & $9,10 \%$ \\
\hline Total & 165 & $100 \%$ \\
\hline
\end{tabular}

Fuente: Producción propia en base a proyecto anteriormente mencionado.

En la tabla $\mathrm{N}^{\circ} 2$, la base son 165 alumnos y no 260 ya que contabilizamos únicamente a aquellos que respondieron afirmativamente a la pregunta. En relación a las respuestas obtenidas, en primer lugar destaca la categoría "Necesidad y nuevo comienzo" con un 38\% de las respuestas. En dicha categoría, agrupamos aquellos discursos que hacían referencia a la necesidad de ayuda que tiene la población que llega a la Argentina, "porque vienen sin nada", "Porque los ayuda en lo que necesita y nos hace más solidarios", entre otras. En general, se trata de discursos que hacen referencia a la falta de recursos que hay en los países de los cuales llegan estas personas, y por lo tanto, la necesidad que tienen aquí de recibir una ayuda para comenzar.

La segunda categoría que se observa es "Personas con igualdad de derechos" con un $36 \%$ de respuestas. En dicha categoría hemos agrupado discursos que hacen alusión al acceso a derechos, sin importar la nacionalidad. Algunos de los discursos fueron "Porque tienen derechos, sean o no de nuestro país son iguales a nosotros", "Porque viven acá y también tienen esos derechos", "Son personas como nosotros, todos fuimos de algún otro país en algún antepasado", entre otras.

En relación a la tercer categoría en porcentaje de respuestas, encontramos "Acepta con restricciones" con un 9\%. En la misma, hemos agrupado aquellos 
discursos que refieren a un supuesto aprovechamiento de parte de los migrantes que reciben esa ayuda, es decir, están de acuerdo con que reciban planes o ayudas del Estado, pero considerando que puede haber situaciones de conveniencia en la recepción de dichas ayudas, que no serían tan necesarias en todos los casos, o bien aceptan que se reciba la ayuda, pero que primero la reciban los nacionales, haciendo referencia a quienes han nacido en el suelo argentino. Algunos ejemplos de dichos discursos son: "Si. Pero en su justa medida. Primero hay que abastecer y ayudar a los más necesitados del país", "si están muy mal económicamente se necesita un impulso, pero tienen que seguir adelante, buscar trabajo", "Sí, pero la prioridad se la deberían dar a los argentinos".

En cuarto lugar, con casi un 5\% de respuestas se encuentra la categoría "Políticamente correcto", donde englobamos aquellas respuestas como "Porque el país sería racista" o "Sería muy discriminatorio". Y finalmente, con tan solo el $2,4 \%$, la categoría "Contribuyen al país", donde agrupamos las respuestas que hacían referencia al aporte que implica la migración para el país, y es por ese motivo, que serían merecedores de ayuda. Entre los discursos se encuentran los siguientes "Porque están trabajando y brindan ayuda al país" o "Ellos también trabajan".

Tal como puede observarse, la mayoría de los alumnos que han respondido afirmativamente a la ayuda social para migrantes, consideran que, por un lado lo necesitan, por venir de países sin recursos y, en segundo lugar, porque existe una representación donde todos deben recibir beneficios y derechos por igual, sin distinción de nacionalidad. En la siguiente tabla analizaremos las respuestas de quienes han respondido de manera negativa, la base de esa tabla son los 100 alumnos que han respondido de esa manera.

Tabla 3 - Estudiantes de escuelas Medias de Villa Lugano según motivo de No acuerdo con que migrantes reciban programas o ayudas sociales. Ciudad Autónoma de Buenos Aires, 2017.

\begin{tabular}{|l|r|r|}
\hline \multicolumn{1}{|c|}{ Motivo } & Frecuencia & Porcentaje \\
\hline Sólo para nacionales & 44 & $44 \%$ \\
\hline Abusadores/aprovechadores & 31 & $31 \%$ \\
\hline No a la ayuda & 9 & $9 \%$ \\
\hline Reciprocidad & 7 & $7 \%$ \\
\hline Ns/Nc & 9 & $9 \%$ \\
\hline Total & 100 & $100 \%$ \\
\hline
\end{tabular}

Fuente: Producción propia en base a proyecto anteriormente mencionado. 
Entre los principales motivos de los estudiantes que se han pronunciado en desacuerdo respecto a que los migrantes reciban ayuda del Estado, se encuentra la categoría que hemos dado en llamar "solo para nacionales". En la misma, hemos agrupado respuestas que, tal como menciona el nombre, se refieren a que la ayuda solo debe ser para Argentinos, por ejemplo, "Tendrían que tener prioridad los argentinos, no tenemos tan buena economía como para darle programas sociales a alguien que ni siquiera es del país" o "el estado tendría que sustentar a la gente que menos tiene con nacionalidad argentina con prioridad", es decir, y en sintonía con lo planteado por Cea D’Ancona (2009) respecto a la idea de la población que los derechos deben primar en los nativos, y especialmente en los contextos de crisis. Es decir, "se defiende el principio de la 'prioridad' en el acceso a los recursos para los 'nacionales”' (CEA D’ANCONA, 2009, p.203).

La segunda categoría en cantidad de respuestas es la que hemos denominado "abusadores/aprovechadores" con el 31\%. En la misma se agrupan discursos que hacen alusión a la falta de voluntad de trabajo de los migrantes, que llegarían a la Argentina para "aprovecharse" de sus recursos. Entre ellos encontramos los siguientes: "Hay gente que no lo necesita y se aprovecha" o "Porque vienen acá solo por eso y a nosotros no nos dan bola y a ellos si por ser de otra nacionalidad".

Si bien se trata de investigaciones enmarcadas en otros universos de estudio, consideramos significativo retomar aquí lo trabajado por Jessica Malegarie (2009, p.66), particularmente en lo que refiere a una mirada prejuiciosa de los nativos sobre la población migrante en la cual "el 'otro' es visto como una amenaza constante ante los bienes, servicios, posesiones y ocupaciones propias". En tal sentido, la autora alude a comentarios tales como "le quitan el trabajo a los argentinos", "utilizan los hospitales públicos quitando los turnos de los argentinos", los cuales marcarían una necesidad de protección de lo propio y de control del "otro", reivindicando y revalorizando de esa manera un "nosotros".

Más recientemente en el tiempo, Néstor Cohen (2014) ha destacado, en su análisis de la institución educativa y el poder judicial, tres núcleos representacionales centrales para la comprensión del modo en que se perciben las relaciones interculturales desde la población receptora y cómo desde esa percepción se constituye un orden que pauta el vínculo con la diversidad. En línea con lo presentado anteriormente, uno de esos núcleos caracteriza a los migrantes externos como portadores de "problemas serios", que ingresan en el espacio de la ilegalidad, donde "nadie controla nada” y están decididos a vender su fuerza de trabajo en condiciones de deslealtad frente a los trabajadores de la sociedad receptora. Ambas referencias, cada una de ellas sustentada con un fuerte compo- 
nente empírico, son prismas significativos para reflexionar en torno al calificativo de "abusadores/aprovechadores".

Otros directamente están en contra de cualquier tipo de ayuda, sin importar la nacionalidad, en dicha categoría encontramos un $9 \%$ de las respuestas. Entre algunos de los discursos se encuentran "Nadie debería tener trato especial" o "Para mi ningún tipo de persona que viva en Argentina tiene que recibir plata regalada"

Finalmente, la categoría "Reciprocidad" con 7\%, allí hemos agrupado aquellas respuestas que dicen no estar de acuerdo con la ayuda porque los argentinos no reciben la misma cuando van a otros países. "Debido a que si nosotros vamos a su país no seríamos ayudados, porque ellos no nos ayudan y no tenemos porqué ayudarlos". Es interesante recuperar, al respecto, aquellas noticias editadas especialmente en febrero del 2018, en torno a un proyecto de Cambiemos para cobrarle a extranjeros el acceso a la salud y la educación; un escenario que nos permite ver las maneras en que, desde distintos ámbitos, circulan discursos en torno a la necesidad de implementar un trato de "reciprocidad" respecto a la inmigración que arriba a Argentina.

\section{Conclusión}

En el presente escrito nos propusimos analizar las representaciones sociales de jóvenes estudiantes que asisten a escuelas públicas de nivel medio en el Barrio de Villa Lugano, respecto a la asignación de ayudas sociales a migrantes por parte del gobierno nacional. Paralelamente, consideramos necesario, como escenario de fondo, dar cuenta de aquellos discursos políticos y modificaciones legislativas relativas a la Ley Migratoria Nacional N²5871 -mediante el Decreto de Necesidad y Urgencia 70/2017- que los mismos antecedieron y, en cierto sentido, habilitaron.

En ese sentido, nos preguntamos acerca de las representaciones que tenían los estudiantes respecto a si acordaban con que el gobierno brindara asistencia social a migrantes y los motivos que sustentaban dichas opiniones, ya sea que estuvieran a favor o en contra del acceso a tal beneficio. Junto a estos interrogantes, nos planteamos describir distintos discursos políticos pronunciados durante la gestión de Cambiemos por parte de funcionarios de primera línea, a partir de la consulta de fuentes periodísticas, particularmente a través de las ediciones digitales de los diarios Clarín y La Nación, que allanaron el camino para la sanción del DNU 70/2017. 
Entendemos que no hay una linealidad entre las representaciones construidas por los estudiantes y los discursos políticos, sino que un modo de concebir la articulación entre ambas líneas de indagación es pensar aquellos discursos a partir de las condiciones de posibilidad que habilitan: más concretamente, en tanto formadores de opinión respecto a la migración, que en los últimos años, y en el marco de lo que consideramos un contexto de conservadurismo político, impactan en la producción y re-producción de determinados universos de representaciones o núcleos representacionales acerca del "otro".

En este sentido, si bien sugerimos que los discursos políticos emergieron como antesala de los cambios a la ley y fueron habilitantes de discursos que circulan en las escuelas, subyace el planteo que las representaciones respecto de la diversidad etnonacional que circula en las aulas por docentes y estudiantes, en tanto sujetos de la sociedad civil atravesados por el sistema escolar, nos permiten dar cuenta de la emergencia de escenarios para que se establezcan determinados tipos de vínculos, tanto al interior como al exterior de los muros de la escuela. En este sentido, sus muros parecieran ser bastante porosos, permeables al contexto en el que está inscrita y, por lo tanto, poco ajena a otros discursos que circulan en la sociedad (MALEGARIE; LANZETTA, 2013). Pretendemos profundizar en futuras indagaciones en las relaciones entre discursos y representaciones, por el momento podemos destacar, al respecto, que existen ciertas afinidades en los modos de caracterizar a la población migrante. Ilustrativamente, al atribuirle "problemas" a los migrantes, desde diferentes ámbitos de la sociedad receptora, ya sean vinculados a la "usurpación" y/o a un presunto vínculo con la delincuencia.

En relación a las respuestas otorgadas por los estudiantes, casi un $40 \%$ de los alumnos encuestados no están de acuerdo con la provisión de ayuda a migrantes en forma de planes sociales o asignaciones por parte del Estado. Gran parte del estudiantado proviene de hogares donde predominan las actividades u ocupaciones de servicios, por tratarse de escuelas ubicadas en un barrio con población de esas características. Sin embargo, la ayuda parece merecerla solo aquel que ha nacido en suelo argentino, sin derecho para aquel que es visto como un "usurpador" de aquellos recursos que solo corresponderían a los argentinos, es decir, aquella población que ha nacido en el país. En un contexto de restricción de recursos, de achicamiento del Estado, los migrantes son vistos como una competencia por los bienes escasos.

Esto cobra mayor relevancia en el contexto en el que escribimos estas reflexiones, donde la pandemia del COVID-19 ha generado que el gobierno nacional dicte el Aislamiento Social Preventivo y Obligatorio (ASPO), que res- 
tringe las posibilidades de generar ingresos a aquellos trabajadores informales, que suelen contarse entre las actividades u ocupaciones de servicios (servicio doméstico, venta ambulante, changas, etc.). Para aquellos que no pudieran percibir ingresos, fue otorgado el Ingreso Familiar de Emergencia (IFE). Sin embargo, solo pueden acceder a él aquellas personas argentinas o bien que cuenten con documentación y dos años de residencia en el país.

Es en este marco que nos preguntamos ¿quiénes tienen acceso legítimo a las ayudas del Estado? ¿Cuáles son las representaciones que se construyen en torno a esta temática en las escuelas? Y, por supuesto, ¿Cuáles serían las respuestas de los alumnos en caso de preguntarse en la actualidad quienes son merecedores del IFE en el actual contexto de ASPO donde son los migrantes una de las poblaciones más vulnerables?

\section{REFERENCIAS}

ABIUSO, F. L. Feos, sucios y malos: un análisis comparativo de imaginarios estatales pasados y presentes acerca de las relaciones entre inmigración y delincuencia en el ámbito de la Ciudad de Buenos Aires. 2020. 231f. Tesis (Doctorado en Ciencias Sociales) - Facultad de Ciencias Sociales, Universidad de Buenos Aires, Buenos Aires, 2020 .

AIMAR, V.; GONZÁleZ, G.; MONTERO, A.; SOZZO, M. Política, policía y violencia en la provincia de Santa Fe. In: SOZZO, M. (dir.). Policía, violencia, democracia: Ensayos sociológicos. Santa Fe: Universidad Nacional del Litoral, 2005. p.15-84.

BEHERAN, M. Tratamientos a la población inmigrante en escuelas de nivel medio de Buenos Aires. Ánfora, Manizales, v.19, n.32, p.49-68, ene-jun. 2012.

BRANDARIZ GARCÍA, J. Á.; DUFRAIX, R.; QUINTEROS, D. La expulsión judicial en el sistema penal chileno: ¿Hacia un modelo de Crimmigration? Política criminal, Santiago, v.13, n.26, p.739-770, 2018.

CAGGIANO, S. Imaginarios racializados y clasificación social: retos para el análisis cultural (y pistas para evitar una deriva decolonial esencialista). Cuadernos Inter.c.a.Mbio Sobre Centroamérica y El Caribe, San José, v.12, n.2, p.157-188, nov. 2015.

CAGGIANO, S. Lo que no entra en el crisol: Inmigración boliviana, comunicación intercultural y procesos identitarios. Buenos Aires: Prometeo Libros, 2005. 
CANELO, B. Espacios disputados: Migrantes y agentes estatales ante el Parque Indoamericano. Revista Ciencias Sociales, Buenos Aires, v.88, p.62-67, 2015.

CANELO, B.; GAVAZZO, N.; NEJAMKIS, L. Nuevas (viejas) políticas migratorias en la Argentina del cambio. Si Somos Americanos, Santiago, v.18, n.1, p.150-182, 2018.

CEA D’ANCONA, M.A. La compleja detección del racismo y la xenofobia a través de encuesta. Un paso adelante en su medición (The Complex Detection of Racism and Xenophobia through Survey Methods. A Step Forward in Their Measurement). Revista Española de Investigaciones Sociológicas (Reis), Madrid, n.125, p.13-45, 2009.

CEA D’ANCONA, M.A.; VALLES, M. Xenofobias y xenofilias en clave biográfica. Madrid: Siglo XXI Editores, 2010.

COHEN, N. Preludio: Los núcleos representacionales constituyentes de la mirada hacia el otro. Unidad Sociológica, Buenos Aires, v.1, n.1, p.6-10, may-sept. 2014.

COHEN, N. Profetizando al diferente. Controversias y Concurrencias Latinoamericanas, Ciudad de México, v.4, n.6, p.187-204, dic. 2012.

COHEN, N. Una interpretación de la desigualdad desde la diversidad étnica. In: COHEN, N. (comp.). Representaciones de la diversidad: trabajo, escuela y juventud. Buenos Aires: Ediciones Cooperativas, 2009. p.11-29.

COHEN, N. El rol del Estado ante las migraciones recientes desde la perspectiva de la población nativa. In: COHEN, N.; MERA, C. (comp.). Relaciones interculturales: experiencias y representación social de los migrantes. Buenos Aires: Antropofagia, 2005. p.141-155.

COHEN, N.; GÓMEZ ROJAS, G. Metodología de la investigación, ¿para qué?: La producción de los datos y los diseños. Buenos Aires: Teseo, 2019.

DE GIORGI, A. El gobierno de la excedencia: postfordismo y control de la multitud. Madrid: Traficante de Sueños, 2006.

DE GIORGI, A. Tolerancia cero: Estrategias y prácticas de la sociedad de control. Barcelona: Virus Editorial, 2005.

DOMENECH, E. 'Las migraciones son como el agua': Hacia la instauración de políticas de 'control con rostro humano'. Polis, Santiago, n.35, p.1-20, 2013. Disponible en: http://journals.openedition.org/polis/9280. Acceso en: 25 ago. 2021.

DOMENECH, E. La "nueva política migratoria" en la Argentina: las paradojas del Programa "Patria Grande". In: PIZARRO, C. (comp.). Migraciones internacionales 
contemporáneas: estudios para el debate. Buenos Aires: Ediciones CICCUS, 2011a. p.119-141.

DOMENECH, E. Crónica de una 'amenaza' anunciada. Inmigración e 'ilegalidad': visiones de Estado en la Argentina contemporánea. In: FELDMAN-BIANCO, B.; RIVERA SÁNCHEZ, L.; STEFONI, C.; VILLA, M. (comp.). La construcción social del sujeto migrante en América Latina: prácticas, representaciones y categorías. Quito: FLACSO Ecuador / CLACSO / UAH, 2011b. p.31-77.

DOMENECH, E.; MAGLIANO, M.J. Migración e inmigrantes en la Argentina reciente: políticas y discursos de exclusión/inclusión. In: ZABALA ARGUELLES, M. del C. (comp.). Pobreza, Exclusión Social y Discriminación Étnico-Racial En América Latina y El Caribe. Bogotá: CLACSO/Siglo del Hombre Editores, 2008. p.423-448.

FRIGERIO, A. "Negros" y "Blancos" en Buenos Aires: Repensando nuestras categorías raciales. Temas de Patrimonio Cultural, Tandil, n.16, p.77-98, 2006.

GALOPPO, L. Necesidad y urgencia en la protección de los derechos de las personas migrantes El amparo presentado por organizaciones de la sociedad civil ante el DNU 70/2017. Revista Temas de Antropología y Migración, Buenos Aires, v.9, p.143$153,2017$.

GALVANI, M. La marca de la gorra: Un análisis de la Policía Federal. Buenos Aires: Capital Intelectual, 2007.

GARCÍA FERRARI, M. Ladrones conocidos / sospechosos reservados. Identificación policial en Buenos Aires, 1880-1905. Buenos Aires: Prometeo, 2010.

GARLAND, D. Governmentality and the Problem of Crime. Theoretical Criminology, London, v.1, n.2, p.173-214, 1997.

GRIMSON, A.; BIDASEA, K. Hegemonía Cultural y políticas de la diferencia. Buenos Aires: CLACSO, 2013.

GUTTON, P. Lo Puberal. Buenos Aires: Editorial Paidós, 1993.

INDEC. Censo Nacional de Población, Hogares y viviendas. Buenos Aires: Instituto Nacional de Estadística y Censo, 2010.

KESSLER, G. La experiencia escolar fragmentada: Estudiantes y docentes en la escuela media de Buenos Aires. Buenos Aires: IIPE-UNESCO, 2002.

KLEIDERMACHER, G.; LANZETTA, D. Una aproximación a las relaciones interculturales en escuelas secundarias de Villa Lugano a partir del análisis de las representaciones sociales hacia población de origen boliviana en Buenos Aires 
(2015-2018). Autoctonía: Revista de Ciencias Sociales e História, Santiago, v.3, n.2, p.132-158, jul-dic. 2019.

KORNBLIT, A. L. Violencia escolar y climas sociales. Buenos Aires: Biblos, 2008.

MALEGARIE, J. El binomio nativos migrantes... In: COHEN, N. (comp.). Representaciones de la diversidad: trabajo, escuela y juventud. Buenos Aires: Ediciones Cooperativas, 2009. p. 65-79.

MALEGARIE, J.; LANZETTA, D. La escuela ante la gestión de la diversidad. In: JORNADAS DE SOCIOLOGÍA, 10., Buenos Aires. Anales [...], Buenos Aires: Facultad de Ciencias Sociales, Universidad de Buenos Aires, 2013. Disponible en: https://cdsa.aacademica.org/000-038/512.pdf. Acceso en: 25 ago. 2021.

MARGULIS, M. Cultura y discriminación social en la época de la globalización. Revista Nueva Sociedad, Caracas, n.152, p.37-52, 1997.

MARRADI, A.; ARCHENTI, N.; PIOVANI, J.I. Metodología de las ciencias sociales. Buenos Aires: Cengage, 2012.

MARTÍNEZ, L. Niñez, migración y perspectivas de derechos: Una aproximación antropológica en el contexto escolar. 2017. 315f. Tesis (Doctorado en Antropología) Facultad de Filosofía y Letras, Universidad de Buenos Aires, 2017.

MONCLÚS MASÓ, M. La reforma de la Ley de migraciones mediante Decreto de Necesidad y Urgencia: un retroceso en la política de derechos humanos. Revista Argentina de Teoría Jurídica, Buenos Aires, n.18, p.1-14, 2017.

MONCLÚS MASÓ, M.; GARCÍA, M. B. El impacto de las migraciones en la criminalidad en la Argentina: mitos y realidades. In: OIM. El impacto de las migraciones en Argentina. Buenos Aires: OIM, 2012. p.323-365. (Cuadernos migratorios, 2.).

MORA, M. La teoría de las representaciones sociales de Serge Moscovici. Athenea Digital, Bellaterra, n.2, p.1-25, otoño 2002. Disponible en: http://www.raco.cat/ index.php/Athenea/article/viewFile/34106/33945. Acceso en: 25 ago. 2021.

MOSCOVICI, S. El psicoanálisis, su imagen y su público. Buenos Aires: Huemul, 1979 .

NOVARO, G.; DIEZ, M.L. ¿Una inclusión silenciosa o las sutiles formas de discriminación? Reflexiones a propósito de la escolarización de nińos boliviano. In: COURTIS, C.; PACECCA, M.I. (ed.). Discriminaciones étnicas y nacionales: Un diagnóstico participativo. Buenos Aires: Editores del Puerto: Asociación por los Derechos Civiles, 2011. p.37-58. 
¿Qué hacemos con los migrantes? Representaciones de alumnos de escuelas medias de villa lugano y discursos políticos sobre la migración limítrofe. Buenos Aires 2016-2018

NOVICK, S. Migración y políticas públicas: Nuevos escenarios y desafíos. Buenos Aires: Catálogos, 2012.

O’MALLEY, P. Riesgo, neoliberalismo y justicia penal. Buenos Aires: Ad-Hoc, 2006.

PENCHASZADEH, A.P.; GARCÍA, L. Política migratoria y seguridad en Argentina hoy: ¿el paradigma de derechos humanos en jaque?. URVIO: Revista Latinoamericana De Estudios De Seguridad, Ecuador, n.23, p.91-109, dic./may 2018-2019.

PEREIRA, M.A. Delito, seguridad, orden público e 'inmigración limítrofe' en Argentina (1976-1995). REMHU: Revista Interdisciplinar da Mobilidade Humana, Brasilia, v.24, n.46, p.177-191, 2016.

RIVERO SIERRA, F. Formas "tangibles" e "intangibles" de discriminación: Aportes para una formalización teórico conceptual. In: PIZARRO, C. (comp.). Migraciones internacionales contemporáneas: estudios para el debate. Buenos Aires: Ediciones CICCUS, 2011. p.269-292.

RUIZ OLABUÉNAGA, J.L. Metodología de la investigación cualitativa. Bilbao: Universidad de Deusto, 1996.

SEGATO, R. L. La nación y sus Otros: Raza, etnicidad y diversidad religiosa en tiempos de Políticas de la Identidad. Buenos Aires: Prometeo Libros, 2007.

SINISI, L. 'Todavía están bajando del cerro': Condensaciones estigmatizantes de la alteridad en la cotidianeidad escolar. In: CONGRESO VIRTUAL DE ANTROPOLOGÍA Y ARQUEOLOGÍA, 1., [s.l.], Anales [...], [s.l.]: Equipo NAyA (Noticias de Antropología y Arqueología), 1998. Disponible en: https:// centroderecursos.cultura.pe/sites/default/files/rb/pdf/todaviaestanbajandodelcerro. pdf. Acceso en: 25 ago. 2021.

SOZZO, M. La inflación punitiva: Un análisis comparativo de las mutaciones del derecho penal en América Latina (1990-2015). Buenos Aires: FLACSO/Café de las Ciudades, 2017.

SOZZO, M. ¿Contando el delito?: Análisis crítico y comparativo de las encuestas de victimización en la Argentina. Cartapacio de Derecho, Buenos Aires, n.5, 2003. Disponible en: http:/www.cartapacio.edu.ar/ojs/index.php/ctp/article/view/38/22. Acceso en: 25 ago. 2021.

TEDESCO, J.C.; KLEIDERMACHER, G.; GRAZZIOTIN NOSCHANG, P. Un análisis de los cambios en las legislaciones migratorias en Brasil y Argentina: implicaciones para la población de origen senegalés. Revista Brasileira de Historia \& Ciencias Sociales, Rio Grande, v.11, n.22, p.302-330, dic. 2019. 
VALLES, M. Técnicas cualitativas de investigación social: Reflexión metodológica y práctica profesional. Madrid: Síntesis, 1997.

VÉLEZ, F.R.; MALUF, N.A. Después de la negación: El Estado argentino frente al racismo y la discriminación. Cuadernos Del Cendes, Venezuela, v.34, n.95, p.155$182,2017$.

ZAYAT, D. Y para (algunos de) los hombres del mundo que quieran habitar el suelo argentino: El retroceso en la política migratoria. Ab: Revista de abogacía, Buenos Aires, v.1, n.1, p.63-70, 2017.

ZEA, L. Fuentes de la Cultura Latinoamericana. México D. F.: Fondo de cultura económica, 1995.

Recebido em: 18 de agosto de 2020

Aprovado em: 07 de dezembro de 2020 